\title{
SEARCHING ANTIVIRAL DRUGS FOR EBOLA VIRUS FROM PHYTOCONSTITUENTS OF AZADIRACHTA INDICA: APPLICATION OF MOLECULAR MODELING STUDIES
}

\author{
PARASURAMAN $\mathrm{P}^{1,2 *}$, SURESH $\mathrm{R}^{2}$, PERUMAL $\mathrm{P}^{3}$ \\ ${ }^{1}$ Department of , Medicinal Chemistry Research Laboratory, Hillside College of Pharmacy \& Research Centre, Bangalore, Karnataka, \\ India. ${ }^{2}$ Department of Pharmacy, Faculty of Engineering and Technology, Annamalai University, Chidambaram, Tamil Nadu, India. \\ ${ }^{3}$ Department of , Faculty of Pharmacy, AIMST University, Jalan Bedong-Semeling 08100, Bedong, Kedah Darul Aman Malaysia. \\ Email: pvpram@gmail.com
}

Received: 10 March 2017, Revised and Accepted: 13 April 2017

\section{ABSTRACT}

Objective: The current objective of the study is to identify some naturally occurring product from Azadirachta indica and evaluate its binding activity against VP24 protein as Ebola virus (EBOV) target through in silico docking studies. Reported phytoconstituents of $A$. indica were prepared for docking evaluation using brincidofovir as the standard.

Methods: In silico docking studies carried out using grid-based ligand docking with energetics (GLIDE) is a ligand binding program provided by Schrödinger.

Results: These results showed that all the selected phytoconstituents showed binding energy ranging between -7.95 and $-1.54 \mathrm{kcal} / \mathrm{mol}$ when compared with that of the standard $(-6.06 \mathrm{kcal} / \mathrm{mol})$. Naturally occurring products catechin, epicatechin, gallic acid, and nimbolide are potential than the standard brincidofovir, but azadirachtin, margolonone, mahmoodin, isomargolonone, gedunin, margolone, nimbidin, and nimbin have low binding affinity toward target when compared with the standard.

Conclusion: These molecular docking analyses of phytoconstituents of $A$. indica could lead to the further development to identify the potent drugs for the treatment of EBOV.

Keywords: Azadirachta indica, VP42 protein, Ebola virus, In silico docking.

(c) 2017 The Authors. Published by Innovare Academic Sciences Pvt Ltd. This is an open access article under the CC BY license (http://creativecommons. org/licenses/by/4. 0/) DOI: http://dx.doi.org/10.22159/ajpcr.2017.v10i7.17500

\section{INTRODUCTION}

It has been shown that a lot of plant products have demonstrated effective antiviral activity. Due to their low toxicity and possible multi-step mechanism, which means, lesser selective pressure for the emergence of resistant strains natural products has been encouraged for searching new drugs. In addition the majority of the compounds used for the clinical purpose are targeted for a diminutive number of viruses [1]. Ethnopharmacological screenings of the medicinal plants have shown that a vast number of phytochemicals such as alkaloids, anthraquinones, coumarins, flavonoids, polyphenols, tannins, terpenoids, among others, are active against virus [2]. The antiviral activity of the natural compounds may occur by inhibition of one or more steps of viral replication. By direct interaction with the viral particle, they can prevent infection; change of the adsorption venture by official to cell receptors; hindrance of infection infiltration into the host cell, by viewing for pathways of actuation of intracellular signs, and all the more regularly, meddling with various phases of viral replication [3]. High-molecular weight compounds such as sulfated

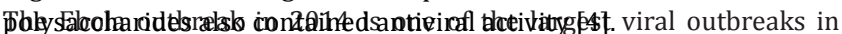
history and the first in West Africa. Ebola virus (EBOV) is a deadly pathogenic virus which is classified under Filoviridae family. In 1976, it was initially reported in the democratic republic of Congo [5,6]. In that capacity, there is no medication or antibody accessible starting at now for the treatment of EBOV disease (http://www.physorg.com/ news134840607.html). EBOV encodes seven polypeptides from its ribonucleic acid (RNA) genome of ca. $19.0 \mathrm{~kb}$, including glycoprotein, nucleoprotein, RNA-subordinate RNA polymerase (L), VP35, VP30, VP40, and VP24 $[7,8]$. Auxiliary network protein VP24 incorporated in EBOV is one of the proteins that assume an essential part in Ebola infection pathology. Furthermore, a few reviews have perceived VP24 as a noteworthy destructiveness factor $[9,10]$. VP24 specifically binds to STAT1. STAT1 is a transcription factor involved in the upregulation of genes because of the signal by various interferons. Out of these proteins, we have concentrated on VP24 protein as the potential medication targets investigating the capacity of these proteins to bring about defensive resistant reactions to EBOV.

Azadirachta indica prominently known as neem has been widely utilized as a part of ayurvedic medication by Indian populace for more than 2000 years. It is utilized generally for the recuperating of different sicknesses. Natural products and their derivatives give an amazing source to new hostile to viral medications [11]. The traditional use as antiviral is portrayed for the treatment of creatures experiencing bovine and avian pox infection diseases by applying a glue of neem leaves specifically on the infected skin [12]. Neem oil can suppress the replication of polio infection, and it can restrain dengue infection type 2 both in vivo and in vitro. Neem bark extract has an immediate hostile to HSV-1 property; it has a high antiviral action of confined polysaccharides from neem leaves against BoHV-1 replication in HEp-2 cells [13-16].

Based on the above view, the aim of our study is to identify some naturally occurring product and evaluate its binding activity against VP24 protein as EBOV target through in silico molecular docking studies.

\section{METHODS}

Glide docking uses the hypothesis of a rigid receptor although scaling of van der Waals radii of nonpolar atoms, which decreases penalties 
for close contacts, can be used to model a slight "give" in the receptor and/or ligand. Docking studies of designed compounds were carried out using grid-based ligand docking with energetics (GLIDE) module version 5.9. Schrödinger, LLC, New York, NY, 2013. The software package running on multiprocessor Linux PC. GLIDE has previously been validated and applied successfully to predict the binding orientation of many ligands.

\section{Protein structure preparation}

The X-ray crystal structures of VP24 (PDB: 4U2X) protein of EBOV retrieved from the RCSB Protein Data Bank. Water molecules of crystallization were removed from the complex, and the protein was optimized for docking using the protein grounding and refinement utility provided by Schrödinger LLC.

\section{Ligand structure preparation}

The structures of energetic constituents of $A$. indica (Fig. 1) were constructed by means of the splinter dictionary of Maestro 9.3 (Schrodinger, LLC) using the optimized potentials for liquid simulationsall atom force field with the steepest descent followed by curtailed Newton conjugate gradient protocol.

\section{Docking protocol}

All docking calculations were performed using the "extra precision" mode of GLIDE program. For the binding site, an assortment of energy grids was premeditated and stored, is distinct in terms of two concentric cubes: The bounding box, which must contain the center of any satisfactory ligand pose, and the enclosing box, which must contain all ligand atoms of an satisfactory pose, with a root mean square deviation of $<0.5 \AA$ and a maximum atomic displacement of $<1.3 \AA$ were eliminated as unneeded to increase assortment in the retained ligand poses. The scale factor for van der Waals radii was applied to those atoms with absolute partial charges $\leq 0.15$ (scale factor of 0.8 ) and 0.25 (scale factor of 1.0) electrons for ligand and protein, respectively. Energy minimization protocol includes dielectric constant of 4.0 and 1000 steps of conjugate gradient. Upon end of each docking calculation, for the most part, 100 poses per ligand were generated. The most excellent docked structure be preferred using a GLIDE score (G-score) function $[17,18]$.

Glide Score $=0.065 * \mathrm{vdW}+0.130 *$ Coul + Lipo + Hbond + Metal + BuryP + RotB + Site

\section{RESULTS AND DISCUSSION}

The docking investigation was completed for the ligands with the target protein VP24 by means of the docking software GLIDE, and the docked images are given away in Figs. 2-7. The structures docked by GLIDE are usually ranked according to the GLIDE scoring function (more negative). The scoring function of GLIDE docking program is offered in the G-score form. The most clear-cut method of evaluating the precision of a docking procedure is to determine how intimately the lowest energy pose (binding conformation) predicted by the object scoring function. To study the molecular basis of interaction and likeness of binding of ligands to VP24 protein, all the ligands were docked into the active site of VP24. The docking result of these ligands is prearranged in Table 1.

The interaction energy includes van der Waals energy, electrostatic energy, as well as intermolecular hydrogen bonding were calculated for each minimized complex. The docking score by means of GLIDE varied from -1.54 to -7.95 against VP24. The GLIDE score for a standard brincidofovir docked with was -6.06 . Naturally occurring products catechin, epicatechin, gallic acid, and nimbolide are potential than the standard brincidofovir and favipiravir, but azadirachtin, margolonone, mahmoodin, isomargolonone, gedunin, margolone, nimbidin, and nimbin have low binding likeness toward the target when compared with the standard. This proves that analogs of catechin, epicatechin, gallic acid, and nimbolide could be impending drugs for the target VP24 in antiviral drug development for EBOV. In generally, for low

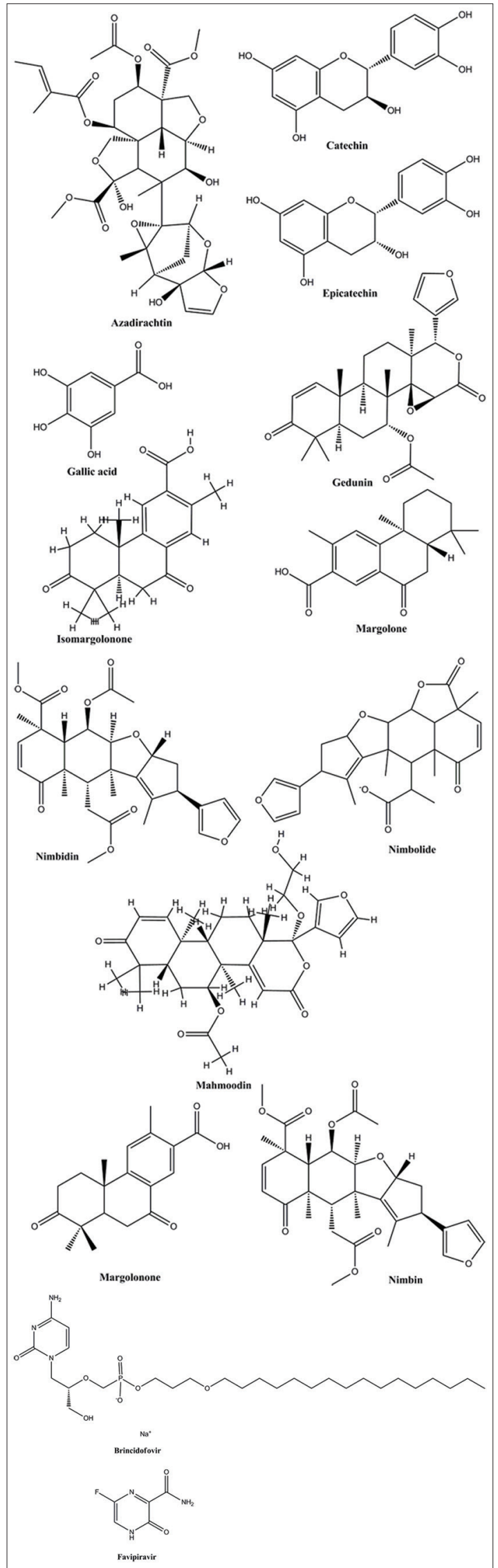

Fig. 1: Ligand structures from Azadirachta indica

GLIDE score, good ligand affinity to the receptor may perhaps expect. Catechin and epicatechin be evidence for the most excellent inhibition 


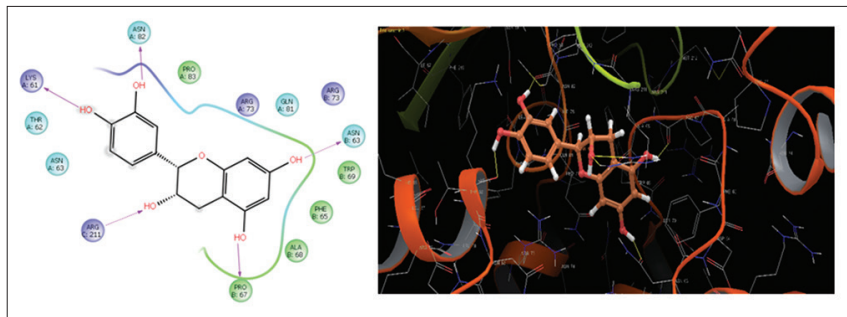

Fig. 2: Ligand interaction of catechin with VP24 (PDB: 4U2X) protein

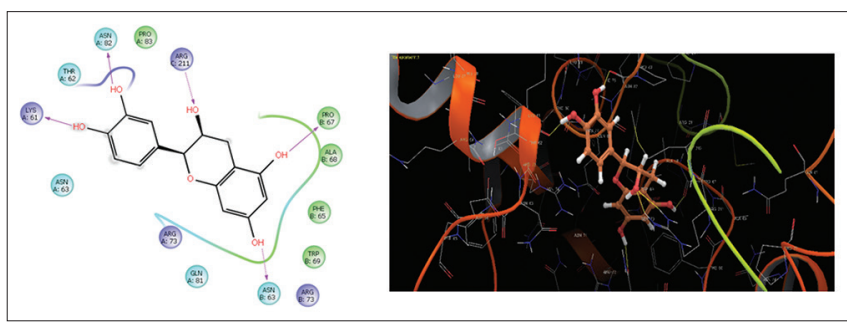

Fig. 3: Ligand interaction of epicatechin with VP24 (PDB: 4U2X) protein

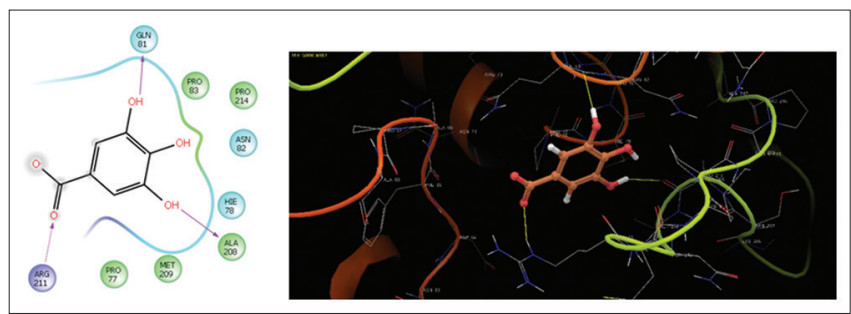

Fig. 4: Ligand interaction of gallic acid with VP24 (PDB: 4U2X) protein

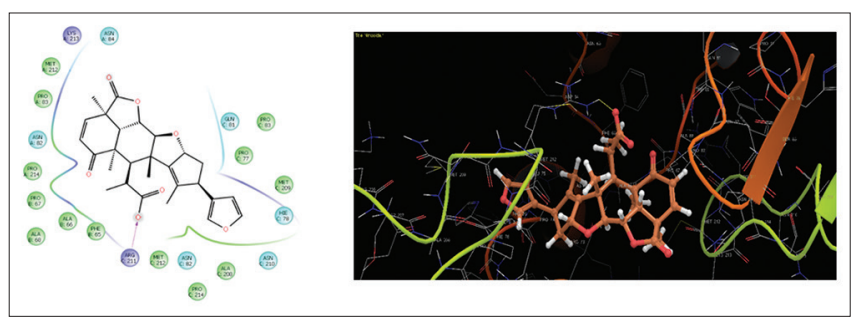

Fig. 5: Ligand interaction of nimbolide with VP24 (PDB: 4U2X) protein

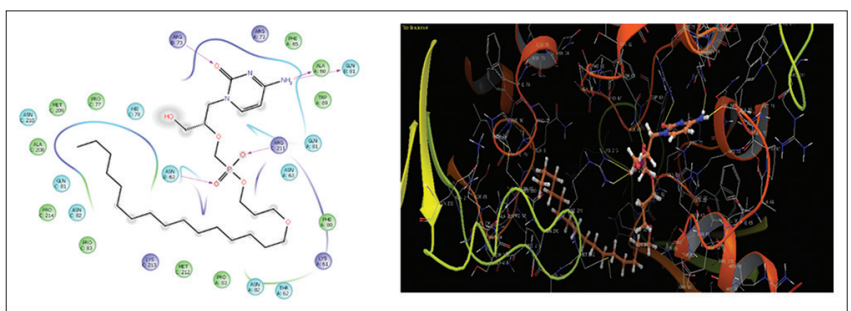

Fig. 6: Ligand interaction of brincidofovir with VP24 (PDB: 4U2X) protein

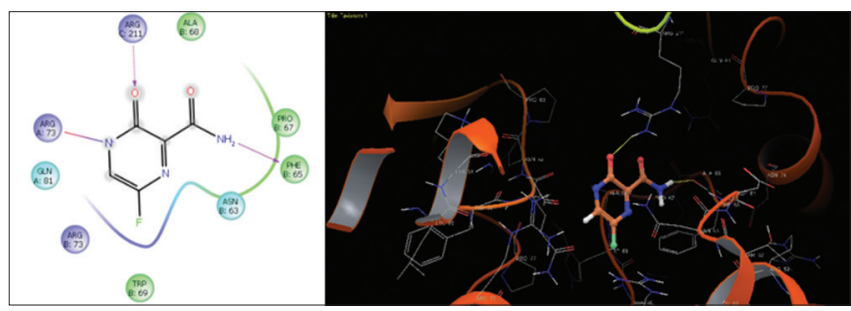

Fig. 7: Ligand interaction of favipiravir with VP24 (PDB: 4U2X) protein
Table 1: Glide score for the docked ligands

\begin{tabular}{lll}
\hline S. No. & Compound & Glide score \\
\hline 1 & Catechin & -7.95 \\
2 & Epicatechin & -7.95 \\
3 & Gallic acid & -6.37 \\
4 & Nimbolide & -6.09 \\
5 & Brincidofovir (standard) & -6.06 \\
6 & Azadirachtin & -5.05 \\
7 & Margolonone & -4.96 \\
8 & Mahmoodin & -4.31 \\
9 & Isomargolonone & -3.74 \\
10 & Favipiravir (Standard) & -3.52 \\
11 & Gedunin & -3.36 \\
12 & Margolone & -3.26 \\
13 & Nimbidin & -1.54 \\
14 & Nimbin & -1.54 \\
\hline
\end{tabular}

for the VP24 with -7.95 glide score against VP24 protein receptor. We found a fantastic agreement between the localization of the inhibitor on docking and from the crystal structure of the protein. Conformational analysis of different docked complexes also shows that residues LYS 61, ASN 63, ARG211, ASN 82, ARG 73, ALA 68, and GLN 81 for VP24 protein play imperative role in this receptor's activity. Docking studies performed by GLIDE have inveterate that above inhibitors fit into the binding pocket of the VP24 receptor. From the results, we may monitor that for successful docking, intermolecular hydrogen bonding and lipophilic interactions between the ligand and the receptor are very significant. The main reason for the increase in GLIDE score is due to the penalties for close intraligand contacts.

CONCLUSION

In conclusion, we have a notorious molecules catechin, epicatechin, gallic acid, and nimbolide, an inventive drug candidate that was docked against VP24 protein in a premeditated attempt to ascertain a new drug candidate, which is able to obstruct with diverse key target points of VP24 in treating EBOV. This compound catechin, epicatechin, gallic acid, and nimbolide well calculated as a best (lead) molecule and we necessitate design analogs, synthesis and evaluate its effectiveness against viral disease caused by EBOV through the molecular level and in vivo studies.

\section{REFERENCES}

1. De Clercq E. Antiviral drugs in current clinical use. J Clin Virol 2004;30(2):115-33

2. Hupfeld J, Efferth T. Review. Drug resistance of human immunodeficiency virus and overcoming it by natural products. In vivo 2009;23(1):1-6.

3. Ghosh T, Chattopadhyay K, Marschall M, Karmakar P, Mandal P, Ray B. Focus on antivirally active sulfated polysaccharides: From structureactivity analysis to clinical evaluation. Glycobiology 2009;19(1):2-15.

4. Ginsberg HS, Goebel WF, Horsfall FL Jr. Inhibition of mumps virus multiplication by a polysaccharide. Proc Soc Exp Biol Med 1947;66(1):99.

5. Branswell H. Ebola war. Sci Am 2015;312:42-9.

6. WHO Ebola Response Team, Aylward B, Barboza P, Bawo L, Bertherat E, Bilivogui P, et al. Ebola virus disease in West Africa - The first 9 months of the epidemic and forward projections. N Engl J Med 2014;371(16):1481-95.

7. Murin CD, Fusco ML, Bornholdt ZA, Qiu X, Olinger GG, Zeitlin L, et al. Structures of protective antibodies reveal sites of vulnerability on Ebola virus. Proc Natl Acad Sci U S A 2014;111(48):17182-7.

8. Kugelman JR, Sanchez-Lockhart M, Andersen KG, Gire S, Park DJ, Sealfon R, et al. Evaluation of the potential impact of Ebola virus genomic drift on the efficacy of sequence-based candidate therapeutics. MBio 2015;6(1):pii:E02227-14.

9. Mateo M, Carbonnelle C, Reynard O, Kolesnikova L, Nemirov K, Page A, et al. VP24 is a molecular determinant of Ebola virus virulence in guinea pigs. J Infect Dis 2011;204 Suppl 3:S1011-20.

10. Reid SP, Leung LW, Hartman AL, Martinez O, Shaw ML, Carbonnelle C, 
et al. Ebola virus VP24 binds karyopherin alpha1 and blocks STAT1 nuclear accumulation. J Virol 2006;80(11):5156-67.

11. Subapriya R, Nagini S. Medicinal properties of neem leaves: A review. Curr Med Chem Anticancer Agents 2005;5(2):149-6.

12. Biswas K, Chattopadhyay I, Banerjee RK, Bandyopadhyay U. Biological activities and medicinal properties of neem (Azadirachta indica). Curr Sci 2002;82:1336-45.

13. Badam L, Joshi SP, Bedekar SS. 'In vitro' antiviral activity of neem (Azadirachta indica. A. Juss) leaf extract against group B coxsackieviruses. J Commun Dis 1999;31(2):79-90

14. Saha S, Galhardi LC, Yamamoto KA, Linhares RE, Bandyopadhyay SS, Sinha S, et al. Water-extracted polysaccharides from Azadirachta indica leaves: Structural features, chemical modification and antibovine herpesvirus Type 1 (BoHV-1) activity. Int J Biol Macromol
2010;47(5):640-5

15. Tiwari V, Darmani NA, Yue BY, Shukla D. In vitro antiviral activity of neem (Azardirachta indica L.) bark extract against herpes simplex virus Type-1 infection. Phytother Res 2010;24(8):1132-40.

16. SaiRam M, Ilavazhagan G, Sharma SK, Dhanraj SA, Suresh B, Parida MM, et al. Anti-microbial activity of a new vaginal contraceptive NIM-76 from neem oil (Azadirachta indica). J Ethnopharmacol 2000;71:377-82.

17. Parasuraman P, Suresh R, Premnath D. Balancing anti-amyloid and anti-cholinesterase capacity in a single chemical entity: In silico drug design. Int J Pharm Pharm Sci 2014;6(2):571-4.

18. Amudha $\mathrm{M}$, Rani S. In silico molecular docking studies on the phytoconstituents of Cadaba fruticosa (L.) Druce for its fertility activity. Asian J Pharm Clin Res 2016;9(2):48-50 